Найденов Алексей Сергеевич

кандидат экономических наук, старший научный сотрудник Института экономики

Уральского отделения РАН

\section{ПОВЫШЕНИЕ ЭФФЕКТИВНОСТИ МЕХАНИЗМОВ ПРОТИВОДЕЙСТВИЯ ТЕНЕВОЙ ЭКОНОМИКЕ В КОНТЕКСТЕ ОБЕСПЕЧЕНИЯ АНТИКРИЗИСНОГО РАЗВИТИЯ РЕГИОНОВ РОССИИ [1]}

Аннотация:

В условиях негативных тенденций развития экономики актуализируются вопросы минимизации отрицательного влияния теневого сегмента и сокращения его размеров как направлений антикризисного управления. В целях формирования мер, нацеленных на повышение эффективности механизмов противодействия теневой экономике, в рамках данной работы представлены результаты изучения теневого сектора и факторов его расиирения в регионах России, а также обобщены итоги международных эмпирических исследований по проблеме выявления детерминант возрастания теневой составляющей в экономике. Международный опыт проанализирован в целях последующей адаптации для использования в отечественной практике. Выделены основные элементы государственной экономической политики, имеющие влияние на динамику и характер теневой экономической деятельности. К числу указанных составляющих относятся меры по противодействию коррупции и экономической преступности; регулирование рынков - финансового, капиталов, труда; регулирование предпринимательской деятельности; налоговая поли тика и ряд других. Сформулированы рекомендации по повышению эффективности механизмов противодействия теневой экономике с учетом факторов неблагоприятной экономической ситуации и кризисных тенденций.

Ключевые слова:

теневая экономика, антикризисное управление, благосостояние, противодействие теневой экономике, экономическая безопасность.
Naidenov Aleksey Sergeyevich

PhD in Economics, Senior Research Fellow, Institute of Economics, Ural branch of the Russian Academy of Sciences

\section{IMPROVING THE EFFECTIVENESS OF MECHANISMS TO COUNTERACT SHADOW ECONOMY IN THE CONTEXT OF ENSURING ANTI-CRISIS DEVELOPMENT OF RUSSIAN REGIONS [1]}

Summary:

In the face of negative trends in the economy, issues of minimizing the negative impact of shadow economy and reducing the shadow sector as one of the areas of crisis management are becoming more relevant. In order to formulate the measures for increasing the effectiveness of mechanisms to counteract shadow economy, the paper presents the results of the study of shadow economy and its growth factors in the regions of Russia and summarizes the results of international empirical studies on the problem of revealing the growth determinants of the shadow component in the economy. This international experience is analyzed for the purpose of subsequent adaptation for its use in Russian practice. As a result, the main components of state economic policy that have an impact on the $d y$ namics and nature of the development of shadow economic activity are identified. These components include the measures to combat corruption and economic crime; regulation of financial, capital, and labor markets; business regulation; tax policy, and a number of other measures. As a result, recommendations for increasing the effectiveness of mechanisms to counter the shadow economy with consideration of factors of an unfavorable economic situation and crisis trends were formulated.

Keywords: shadow economy, crisis management, welfare, counteracting shadow economy, economic security.

Противодействие теневой экономике как одному из факторов дестабилизации является важнейшим из направлений государственной политики: «Теневая экономика может не только оказывать влияние на экономики мира, нанося ущерб экономическому развитию, но и вредить личному благосостоянию граждан... подрывать способность публичной власти поддерживать устойчивый экономический рост» [2, р. 1120]. Как отмечает В.И. Самаруха, «современная теневая экономика максимально разрушительно влияет на легальную экономическую деятельность в стране» [3, с. 207].

В условиях кризисных и негативных тенденций в экономике вопросы противодействия теневому сектору еще более актуализируются. Кроме того, общее отрицательное влияние на экономику имеет его контрциклический характер относительно экономических кризисов [4]: теневой сегмент существенно расширяется в период депрессий и спадов, а потому требует еще более пристального внимания именно в сложное для экономики время. В рамках сформировавшихся в последние годы отрицательных экономических трендов России, обусловленных рядом внешних и внутренних фракторов, противодействие теневой экономике вновь становится актуальным для 
страны в целом и ее регионов в частности. Данный фракт отмечается в многочисленных публикациях на тему текущего состояния экономики РФ, а также в официальных отчетах международных организаций, например Всемирного банка [5].

Вместе с тем, несмотря на существенное количество публикаций по теме теневой экономики и противодействия ей, следует отметить отсутствие целостности в подходах и единства взглядов на роль и характер влияния отдельных социально-экономических факторов на динамику теневой деятельности. Как указывают Р. Гоел и М. Нельсон, отчасти это является следствием проблемы точного измерения величины теневого сектора экономики [6].

Кроме того, в публикациях встречаются различающиеся толкования сущности теневой экономики и ее составляющих, однозначная трактовка которых служит залогом обоснованности методических подходов к исследованию теневой экономики, а также формирования мер по противодействию ей. Мы исходим из общераспространенного определения сущности теневой экономики как совокупности легальных видов деятельности, скрытых от государственного статистического учета и налогообложения [7]. Данный подход отвечает положениям Системы национальных счетов и официальным рекомендациям по измерению ненаблюдаемой экономики [8].

В соответствии с указанной трактовкой нами ранее разработан методический аппарат, который используется в настоящей статье для количественного анализа величины теневого сектора экономики [9]. Полученные оценки применяются в данном исследовании при разработке обоснованных рекомендаций по повышению эффективности механизмов противодействия теневой экономике.

Влияние социально-экономических и институциональных факторов на теневую экономику в субъектах РФ. В рамках исследования проанализированы факторы, воздействующие на динамику теневого сектора в разрезе регионов (в силу существенной социально-экономической дифференциации субъектов РФ). При этом для оценки значимости социально-экономических факторов проведен факторный анализ на основе 89 показателей, сгруппированных в следующие индикативные блоки: внешнеэкономическая деятельность, состояние государственных финансов, состояние хозяйствующих субъектов, инвестиционная и производственная сфреры, рынок товаров и услуг, рынок труда, доходы населения, демографическое состояние, правопорядок.

В результате последующего применения метода главных компонент выделены ключевые агрегированные фракторы, описывающие влияние социально-экономического состояния на динамику теневой активности и определяющие расширение теневого сегмента. В зависимости от анализируемого региона выявлено от 8 до 15 составляющих, объясняющих 82-97 \% всей дисперсии уровня теневой экономики в субъектах РФ.

В результате выделены следующие ключевые факторы, оказывающие наибольшее влияние на динамику теневого сегмента в регионах России за исследуемый период 2006-2018 гг.:

- динамика экономического роста;

- инвестиционная активность (общий объем инвестиций, величина иностранных вложений и другие показатели);

- миграционная активность населения;

- фринансовое состояние банковского сектора;

- уровень налоговой нагрузки;

- доходов населения;

- предпринимательской активности;

- цен (индексы потребительских цен и цен производителей на промышленные товары).

Кроме того, проведен анализ влияния институциональной среды и административных барьеров на объемы теневой экономики в субъектах РФ в 2012-2018 гг. В результате выявлено, что в отличие от многих стран (в первую очередь относящихся к категории развитых) для России характерна низкая величина эластичности теневой активности в зависимости от состояния институциональной среды и относительного уровня административных барьеров: 0,30 отн. ед. в сравнении с 0,60-1,00 отн. ед. в развитых государствах (США - 0,88, Германия - 0,98, Австрия - 1,50 и т. д.).

Вместе с тем важным итогом исследования является тот факт, что если в целом России свойственна низкая эластичность теневой экономики в зависимости от факторов институциональной среды, то в экономически развитых регионах РФ ситуация обратная - эластичность превышает 1,00 отн. ед., а в некоторых регионах - достигает 2,00 отн. ед. Например, в Москве она составляет 2,11 отн. ед., Санкт-Петербурге - 2,03, Республике Татарстан - 1,92, Свердловской области - 1,97 отн. ед.

Таким образом, ослабление административных барьеров и улучшение институциональной среды являются приоритетными мерами не для всех регионов России в части противодействия теневой экономике. В данной области требуется дифференциация используемых подходов: для 
некоторых субъектов РФ в первую очередь необходимо сосредоточиться на преодолении накопленных институциональных проблем, для других - на решении вопросов достижения устойчивого роста и ускорения экономического развития.

Мировой опыт исследования влияния факторов роста теневой экономики. В рамках данной работы предлагается обратиться к международным исследованиям в целях формирования более универсальных рекомендаций по противодействию теневой экономике. Необходимо рассмотреть результаты количественного анализа на основе статистических сведений и эконометрических моделей. Обобщенный международный опыт используется в целях последующей адаптации для реализации в российской практике.

Рассматривая детерминанты распространения теневой экономики, противодействие которым способствует снижению ее объемов, можно отметить результаты исследований Р. Гоела и М. Нельсона, согласно которым чрезмерное административное регулирование является более значимым фактором для расширения теневого сегмента, нежели какие-либо иные. Например, «повышение показателя сложности уплаты налогов на одно стандартное отклонение приводит к увеличению теневой экономики в среднем на $10 \%$... аналогичное повышение в области стоимости создания бизнеса приводит к двукратному возрастанию» [10].

На основе моделирования установлено, что уменьшение рыночного регулирования приводит к соответствующему снижению теневой активности [11]. Аналогичная ситуация наблюдается при управлении рынком труда, в частности речь идет о «стоимости» официального трудоустройства налогах, затратах на наем, сертификацию и т. д. Таким образом, в целях противодействия теневой экономике требуется снижение подобной стоимости [12]. На высокую значимость фракторов, связанных со сферой занятости, обращают внимание и другие исследователи, что обусловливает включение трудового регулирования в перечень мер по противодействию теневому сектору [13].

Вместе с тем не стоит забывать, что снижение степени регулирования не должно приводить к ликвидации контроля и устранению наказания за ведение теневой деятельности. Принятие мер административного характера, направленных на создание условий неотвратимости наказания за подобного рода активность, является не менее важным механизмом противодействия теневой экономике. Более того, учитывая высокую взаимосвязь уровней коррупции и теневого сектора, значимым элементом устранения теневых форм хозяйствования выступает тотальное противостояние любым формам коррупции. Это особенно актуально в целях антикризисного управления по причине ограниченности экономических ресурсов.

Данные тезисы находят подтверждение в исследовании Л. Батрансы с соавторами [14], проведенном на основе эконометрического анализа 193 стран. Согласно мнению этих ученых, теневая экономика и коррупция абсолютно комплементарны - повышение или снижение объемов теневого сегмента приводит к аналогичному эффректу для уровня коррупции. К аналогичным выводам пришли другие авторы в результате эконометрической оценки теневой экономики в государствах Европейского союза [15].

При этом главный упор необходимо делать не столько на тяжесть наказания, сколько на его неотвратимость и глубокую институционализацию в экономической жизни. Как отмечает И. Баяр [16], верховенство закона имеет крепкую статистическую связь с уменьшением доли теневого сектора в структуре экономики. В связи с этим данное направление должно быть ключевым при формировании механизмов противодействия теневой экономике.

В целом следует обозначить, что улучшение институциональной среды является важным фрактором снижения уровня теневой экономики и сопутствующих ей негативных социально-экономических последствий. Так, согласно результатам исследования румынских ученых [17], относительный объем теневого сектора меньше в странах с более стабильной политической системой и гарантированными гражданскими свободами. Авторы указанной работы демонстрируют статистическую значимость вложений в человеческий капитал в целях сокращения сегмента теневой экономики. Государства, где забота о гражданах, включая в первую очередь развитие здравоохранения и образования, находится на достаточно высоком уровне, вовлеченность населения в теневую деятельность существенно ниже.

Следует обратить внимание на то, что повышение уровня благосостояния граждан служит фрактором уменьшения теневой активности [18]. Согласно количественным оценкам влияния неравенства доходов на объемы теневой экономики - увеличение коэфффициента Джини на 1,0\% приводит к расширению теневого сектора в среднем на 0,2-0,3 \% [19]. Более того, существует явление замкнутого круга, при котором высокий уровень теневой экономики обусловливает возникновение «ловушки бедности», когда компании уходят в тень и остаются малым бизнесом, не имея стимулов развиваться в крупные официальные предприятия [20]. 
В связи с этим, как отмечает С. Тумэн, существенное влияние на сокращение объемов теневой экономики оказывает механизм перераспределения благосостояния населения, в первую очередь снижение уровня налогообложения для малого бизнеса [21]. В части реализации механизмов перераспределения доходов эффективной мерой являются целевые социальные выплаты малообеспеченным гражданам [22]. В данном контексте представляются удачными эксперимент по внедрению налогообложения для самозанятых, а также распространение этого опыта за пределы пилотных регионов России - Москвы, Московской и Калужской областей, Республики Татарстан.

C фактором благосостояния связано различие в уровне теневой экономики в развитых и развивающихся странах, прочие условия по-разному влияют на теневой сегмент в указанных группах государств. В частности, если «некоторые факторы, такие как высокая инфляция или большая налоговая нагрузка, способствуют росту теневой экономики для всех стран, то некоторые регуляторные барьеры (например, процедуры для старта бизнеса) стимулируют теневую активность в основном в развивающихся странах» [23, р. 361].

Другое направление - максимальное упрощение и дерегуляция, особенно в части налогового администрирования. В связи с этим представляется уместным как минимум сокращение транзакционных издержек взаимодействия с государством путем всесторонней цифровизации, что, с одной стороны, снизит стоимость процесса, с другой - повысит его прозрачность.

К числу других экономических фракторов, способствующих уменьшению объемов теневой экономики, относится достаточное количество доступных источников фринансирования - их дефицит или высокая стоимость в официальном секторе экономики обусловливает возникновение теневых ресурсов финансирования компаний, в том числе долгосрочных инвестиций, что впоследствии приводит к транзиту денежных потоков из официального сегмента в теневой [24]. В связи с этим в рамках антикризисного развития регионов России и повышения эффективности механизмов противодействия теневой экономике требуется уделять особое внимание финансовой политике.

Многие авторы акцентируют внимание на том, что, несмотря на первоочередную роль экономических мер в уменьшении объемов теневой экономики, важно помнить и о существенном влиянии фрактора снижения административных барьеров [25]. Чем меньше возможностей у административного аппарата для установки всевозможных ограничений для ведения предпринимательской и хозяйственной деятельности, тем ниже уровень коррупции и, соответственно, теневой экономики. Как отмечают Р. Гоел и М. Нельсон, «сложность процедур, особенно в части количества действий, необходимых для уплаты налогов (нежели уровень налоговой нагрузки), является драйвером ухода в тень для бизнеса» [26, р. 361].

К числу других институциональных фракторов, оказывающих влияние на теневую экономику, относится уровень доверия в обществе, который определяет степень зарегулированности экономической деятельности и взаимосвязан с уровнем распространенности теневых форм хозяйствования в экономике [27]. Кроме того, одним из условий эфффективного противодействия теневому сектору является целевой характер принимаемых мер, что подразумевает в том числе разное влияние одних и тех же факторов на склонность к ведению теневого бизнеса уже работающими и только создаваемыми предприятиями, а также межстрановые и межрегиональные различия [28]. Например, для развитых и развивающихся государств рецепты противодействия различаются, как и для разных регионов.

При этом Р. Гоел и М. Нельсон считают целесообразным разделение мер административного воздействия в рамках противодействия теневой экономике для компаний, которые уже работают на рынке и имеют склонность к переходу в неофициальный сектор, и организаций, только нацеливающихся на теневой сегмент. Подобная диверсификация может быть выполнена в форме уменьшения налоговой нагрузки для действующих предприятий и снижения административных барьеров входа на рынок для фирм, начинающих хозяйственную деятельность. Данные методологические принципы рекомендуется включать в основу мер по снижению объемов теневой экономики.

Таким образом, в статье представлены итоги изучения способов повышения эффективности механизмов противодействия теневой экономике на базе обобщения результатов двух векторов исследования: анализа международных работ, посвященных выявлению детерминант расширения теневого сектора, и авторской количественной оценки влияния социально-экономических и институциональных факторов на динамику теневой экономики в регионах России. Показано, что, поскольку одним из ключевых условий развития теневой деятельности (как в России, так и за рубежом) служит низкий уровень доходов, уменьшающийся в период экономических кризисов, приоритетным направлением противодействия теневому сегменту в подобной ситуации выступает стимулирование экономического роста.

Акцентировано внимание на значительной роли уровня благосостояния и государственных расходов на развитие человеческого капитала (здравоохранение и образование). В условиях экономических кризисов и спадов, несмотря на сокращение бюджетных доходов, принципиально 
важным является сохранение объемов вложений в эти отрасли экономики на текущем уровне или даже их увеличение. Данное направление должно стать одним из ключевых в планировании государственных расходов в России, особенно с учетом бюджетных дисбалансов в регионах, а также систематического недофинансирования таких областей, как здравоохранение и образование. В связи с этим актуализируется необходимость проведения исследований теневой экономики на региональном уровне с последующим внедрением мер государственной политики по противодействию теневой деятельности с учетом региональной специфики субъектов РФ.

Важность снижения административных барьеров и упрощения регулирования различных рынков подтверждается как международными сравнительными исследованиями, так и результатами изучения регионов России. Например, при оценке роли трудового регулирования в динамике теневой экономики в России и ее регионах необходимо акцентировать внимание на значимости пенсионного реформирования. Увеличение срока выхода на пенсию при недостаточной эффективности пенсионной системы (в части соотношения отчислений на пенсионное обеспечение работающих граждан и величины пенсионных выплат) является фрактором возрастания «издержек» деятельности в официальном секторе экономики. При этом отсутствие продуктивных мер по стимулированию рынка труда в неблагоприятных регионах РФ (различающихся в экономическом, климатическом, географическом и иных планах) способствует расширению теневого сектора.

Сравнительный анализ показал важность институциональных факторов, в том числе доверия населения. В рамках политики противодействия теневой экономике в РФ повышение индекса доверия является важным направлением в силу его недостаточности [29]. Россия в настоящее время относится к группе стран с его низким уровнем.

Установлена значимость противодействия коррупции как фактора развития теневой экономики, а также сокращения государственного аппарата и уменьшения его роли в экономической жизни общества [30]. Это особенно актуально для России, занимавшей в 2017 г. 93-е место в рейтинге государственной эффрективности.

Результаты международных исследований позволили уточнить методологические принципы, связанные с дифференциацией мер по противодействию теневой экономике, в зависимости от текущего состояния конкретной территории. Указанные направления государственной политики являются ключевыми для обеспечения антикризисного развития регионов России в силу высокой значимости минимизации негативного влияния теневого сектора на национальную экономику в периоды экономических спадов.

\section{Ссылки и примечания:}

1. Статья выполнена в соответствии с планом НИР ФГБУН Института экономики УрО РАН на 2019-2021 гг.

2. The Strenght of the Relationship Between Shadow Economy and Corruption: Evidence from a Worldwide Country-Sample / L. Batrancea, A. Nichita, I. Batrancea, L. Gaban // Social Indicators Research. 2018. Vol. 138, iss. 3. P. 1119-1143. https://doi.org/10.1007/s11205-017-1696-z.

3. Самаруха В.И., Самаруха А.В., Самаруха И.В. Проблемы противодействия налоговым преступлениям // Всероссийский криминологический журнал. 2018. Т. 12, № 2. С. 199-210. https://doi.org/10.17150/2500-4255.2018.12(2).199-210.

4. Colombo E., Onnis L., Tirelli P. Shadow Economies at Times of Banking Crises: Empirics and Theory // Journal of Banking and Finance. 2016. Vol. 62. P. 180-190. https://doi.org/10.1016/j.jbankfin.2014.09.017 ; Elgin C. Cyclicality of Shadow Economy // Economic Papers: A Journal of Applied Economics and Policy. 2012. Vol. 31, iss. 4. P. 478-490. https://doi.org/10.1111/1759-3441.12011.

5. Умеренные темпы роста экономики; в центре внимания - неформальный сектор [Электронный ресурс] : доклад об экономике России № 41 // The World Bank. 2018. URL: http://pubdocs.worldbank.org/en/908421560108417755/rer-41russian.pdf (дата обращения: 03.12.2019).

6. Goel R.K., Nelson M.A. Shining a Light on the Shadows: Identifying Robust Determinants of the Shadow Economy // Economic Modelling. 2016. Vol. 58. P. 351-364. https://doi.org/10.1016/j.econmod.2016.06.009.

7. Tolla M. Individual Approach to the Shadow Economy // Journal of Interdisciplinary Economics. 2017. Vol. 29, iss. 2. P. $153-175$. https://doi.org/10.1177/0260107917709169.

8. Измерение ненаблюдаемой экономики : руководство. М., 2003. 295 с.

9. Найденов А.С. Роль налогового администрирования в противодействии теневой экономической деятельности. Екатеринбург, 2012. 182 c.

10. Goel R.K., Nelson M.A. Op. cit.

11. Charlot O., Malherbet F., Terra C. Informality in Developing Economies: Regulation and Fiscal Policies // Journal of Economic Dynamics and Control. 2015. Vol. 51. P. 1-27. https://doi.org/10.1016/j.jedc.2014.09.031.

12. Capasso S., Jappelli T. Financial Development and the Underground Economy // Journal of Development Economics. 2013. Vol. 101. P. 167-178. https://doi.org/10.1016/j.jdeveco.2012.10.005

13. Blanton R.G., Peksen D. Labor Laws and Shadow Economies: A Cross-National Assessment // Social Science Quarterly. 2019. Vol. 100, iss. 5. P. 1540-1565. https://doi.org/10.1111/ssqu.12685; Identification of the Shadow Economy Determinants for the Eurozone Member States: Application of the Mimic Model / R. Remeikiené, L. Gasparènienè, V. Chadyšas, M. Cepel // Journal of Business Economics and Management. 2018. Vol. 19, iss. 6. P. 777-796. https://doi.org/10.3846/jbem.2018.6276.

14. The Strenght of the Relationship ...

15. Corruption and Shadow Economy in Transition Economies of European Union Countries: a Panel Cointegration and Causality Analysis / Yi. Bayar, H. Odabas, M.U. Sasmaz, O.F. Ozturk // Economic Research. 2018. Vol. 31, iss. 1. P. 1940-1952. https://doi.org/10.1080/1331677X.2018.1498010. 
16. Ibid.

17. The Strenght of the Relationship ...

18. Dell'Anno R. Analyzing the Determinants of the Shadow Economy with a "Separate Approach". An Application of the Relationship Between Inequality and the Shadow Economy // World Development. 2016. Vol. 84. P. 342-356. https://doi.org/10.1016/j.worlddev.2015.08.026 ; Goel R.K., Nelson M.A. Op. cit.

19. Berger W., Salotti S., Sardà J. Do Fiscal Decentralization and Income Inequality Affect the Size of the Shadow Economy? A Panel Data Analysis for OECD Countries // Applied Economics Letters. 2018. Vol. 25, iss. 8. P. 571-575. https://doi.org/10.1080/13504851.2017.1346356.

20. Tumen S. Entrepreneurship in the Shadows: Wealth Constraints and Government Policy // Economics of Transition. 2017. Vol. 25, no. 2. P. 239-270.

21. Ibid.

22. Berger W., Salotti S., Sarda J. Op. cit.

23. Goel R.K., Nelson M.A. Op. cit. P. 361

24. Blackburn K., Bose N., Capasso S. Tax Evasion, the Underground Economy and Financial Development // Journal of Economic Behavior and Organization. 2012. Vol. 83, iss. 2. P. 243-253. https://doi.org/10.1016/j.jebo.2012.05.019 ; Capasso S., Jappelli T. Op. cit.

25. The Strenght of the Relationship ...

26. Goel R.K., Nelson M.A. Op. cit. P. 361.

27. Ibid.

28. Ibid.

29. Ibid.

30. Berger W., Salotti S., Sarda J. Op. cit.

\section{References:}

Batrancea, L, Nichita, A, Batrancea, I \& Gaban, L 2018, 'The Strength of the Relationship Between Shadow Economy and Corruption: Evidence from a Worldwide Country-Sample', Social Indicators Research, vol. 138, iss. 3, pp. 1119-1143, https://doi.org/10.1007/s11205-017-1696-z.

Bayar, Yi, Odabas, H, Sasmaz, MU \& Ozturk, OF 2018, 'Corruption and Shadow Economy in Transition Economies of European Union Countries: a Panel Cointegration and Causality Analysis', Economic Research, vol. 31, iss. 1, pp. 1940-1952, https://doi.org/10.1080/1331677X.2018.1498010.

Berger, W, Salotti, S \& Sarda, J 2018, 'Do Fiscal Decentralization and Income Inequality Affect the Size of the Shadow Economy? A Panel Data Analysis for OECD Countries', Applied Economics Letters, vol. 25, iss. 8, pp. 571-575, https://doi.org/10.1080/13504851.2017.1346356.

Blackburn, K, Bose, N \& Capasso, S 2012, 'Tax Evasion, the Underground Economy and Financial Development', Journal of Economic Behavior and Organization, vol. 83, iss. 2, pp. 243-253, https://doi.org/10.1016/j.jebo.2012.05.019.

Blanton, RG \& Peksen, D 2019, 'Labor Laws and Shadow Economies: A Cross-National Assessment', Social Science Quarterly, vol. 100, iss. 5, pp. 1540-1565, https://doi.org/10.1111/ssqu.12685.

Capasso, S \& Jappelli, T 2013, 'Financial Development and the Underground Economy', Journal of Development Economics, vol. 101, pp. 167-178, https://doi.org/10.1016/j.jdeveco.2012.10.005.

Charlot, O, Malherbet, F \& Terra, C 2015, 'Informality in Developing Economies: Regulation and Fiscal Policies', Journal of Economic Dynamics and Control, vol. 51, pp. 1-27, https://doi.org/10.1016/j.jedc.2014.09.031.

Colombo, E, Onnis, L \& Tirelli, P 2016, 'Shadow Economies at Times of Banking Crises: Empirics and Theory', Journal of Banking and Finance, vol. 62, pp. 180-190, https://doi.org/10.1016/j.jbankfin.2014.09.017.

Dell'Anno, R 2016, 'Analyzing the Determinants of the Shadow Economy with a "Separate Approach". An Application of the Relationship Between Inequality and the Shadow Economy', World Development, vol. 84, pp. 342-356, https://doi.org/10.1016/j.worlddev.2015.08.026.

Elgin, C 2012, 'Cyclicality of Shadow Economy', Economic Papers: A Journal of Applied Economics and Policy, vol. 31, iss. 4, pp. 478-490, https://doi.org/10.1111/1759-3441.12011.

Goel, RK \& Nelson, MA 2016, 'Shining a Light on the Shadows: Identifying Robust Determinants of the Shadow Economy', Economic Modelling, vol. 58, pp. 351-364, https://doi.org/10.1016/j.econmod.2016.06.009.

Measuring the Non-Observed Economy: a Handbook 2003, Moscow, 295 p., (in Russian).

Moderate Economic Growth Rate; Focus on Informal Sector: a Report on Russian Economy No. 412018,63 p., viewed 03 December 2019, <http://pubdocs.worldbank.org/en/908421560108417755/rer-41-russian.pdf>, (in Russian).

Naydenov, AS 2012, The Role of Tax Regulation in Counteracting Shadow Economic Activity, Ekaterinburg, 182 p., (in Russian).

Remeikienè, R, Gasparènienè, L, Chadyšas, V \& Cepel, M 2018, 'Identification of the Shadow Economy Determinants for the Eurozone Member States: Application of the Mimic Model', Journal of Business Economics and Management, vol. 19, iss. 6, pp. 777-796, https://doi.org/10.3846/jbem.2018.6276.

Samarukha, VI, Samarukha, AV \& Samarukha, IV 2018, 'Problems of Counteracting Tax Crimes', Vserossiysky kriminologichesky zhurnal, vol. 12, no. 2, pp. 199-210, https://doi.org/10.17150/2500-4255.2018.12(2).199-210, (in Russian).

Tolla, M 2017, 'Individual Approach to the Shadow Economy', Journal of Interdisciplinary Economics, vol. 29, iss. 2, pp. 153-175, https://doi.org/10.1177/0260107917709169.

Tumen, S 2017, 'Entrepreneurship in the Shadows: Wealth Constraints and Government Policy', Economics of Transition, vol. 25 , no. 2, pp. 239-270.

Редактор: Тюлюкова Мария Олеговна Переводчик: Жбан Екатерина Сергеевна 